\title{
Early Exposure to Alcohol Leads to Permanent Impairment of Dendritic Excitability in Neocortical Pyramidal Neurons
}

\author{
Alberto Granato, ${ }^{1}$ Lucy M. Palmer, ${ }^{2}$ Andrea De Giorgio, ${ }^{1}$ Daniela Tavian, ${ }^{1}$ and Matthew E. Larkum ${ }^{2}$ \\ ${ }^{1}$ Department of Psychology, Catholic University, 20123 Milan, Italy, and ${ }^{2}$ Institute of Physiology, University of Bern, CH-3012 Bern, Switzerland
}

\begin{abstract}
Exposure to alcohol in utero is a well known cause of mental retardation in humans. Using experimental models of fetal alcohol spectrum disorder, it has been demonstrated that cortical pyramidal neurons and their projections are profoundly and permanently impaired. Yet, how the functional features of these cells are modified and how such modifications impact cognitive processes is still unknown. To address this, we studied the intrinsic electrophysiological properties of pyramidal neurons in young adult rats (P30-P60) exposed to ethanol inhalation during the first week of postnatal life (P2-P6). Dual whole-cell recordings from the soma and distal apical dendrites were performed and, following the injection of depolarizing current into the dendrites, layer 5 neurons from ethanol-treated (Et) animals displayed a lower number and a shorter duration of dendritic spikes, attributable to a downregulation of calcium electrogenesis. As a consequence, the mean number of action potentials recorded at the soma after dendritic current injection was also lower in Et animals. No significant differences between Et and controls were observed in the firing pattern elicited in layer 5 neurons by steps of depolarizing somatic current, even though the firing rate was significantly lower in Et animals. The firing pattern and the firing rate of layer $2 / 3$ neurons were not affected by alcohol exposure.
\end{abstract}

\section{Introduction}

The effects of in utero alcohol exposure in humans are globally referred to as fetal alcohol spectrum disorder (FASD) and represent one of the chief causes of mental retardation. Experimental models of FASD have made it possible to investigate the main phenomena leading to the deranged brain organization after early exposure to ethanol. The cerebral cortex is one of the brain regions most affected by FASD, and its alterations account for most of the observed cognitive defects (Guerri et al., 2009). When exposed to alcohol, developing cortical neurons are subjected to an increased rate of apoptosis (Ikonomidou et al., 2000; Mooney and Miller, 2001) and the surviving cortical neurons undergo a permanent rearrangement of their morphology and connections (Miller et al., 1990; Granato et al., 2003). Although such a rewiring of the cortical network is likely to affect its functional features, little is known about how the electrophysiological behavior of cortical neurons is modified. Early alcohol exposure results in persistent deficits of the fine functional organization (Xie et al., 2010 ) and plasticity (Medina and Krahe, 2008) of primary sensory cortices. Since the intrinsic properties of cortical neurons are key determinants of cortical plasticity (Letzkus et al., 2006), we decided to study the electrophysiological features of pyramidal neurons in the somatosensory cortex of young adult rats (P30-

Received Nov. 2, 2011; revised Nov. 29, 2011; accepted Dec. 9, 2011.

Author contributions: A.G. and M.E.L. designed research; A.G., L.M.P., A.D.G., D.T., and M.E.L. performed research; A.G., A.D.G., D.T., and M.E.L. analyzed data; A.G., L.M.P., and M.E.L. wrote the paper.

This work was supported by MIUR (Italian Ministry of Research), SystemsX.ch (NeuroChoice), and Swiss National Science Foundation (PPO0A-102721).

Correspondence should be addressed to Dr. Alberto Granato, Department of Psychology, Catholic University, Largo A. Gemelli 1, 20123 Milan, Italy. E-mail: alberto.granato@unicatt.it.

DOI:10.1523/JNEUROSCI.5520-11.2012

Copyright $\odot 2012$ the authors $\quad 0270-6474 / 12 / 321377-06 \$ 15.00 / 0$
P60) exposed to ethanol during the first postnatal week. Due to the immaturity of the brain at birth (Dobbing and Sands, 1979), the early postnatal exposure to ethanol reproduces several aspects of fetal alcohol effects in humans. Using simultaneous recording from the soma and apical dendrite, we demonstrate that dendrites of layer 5 neurons are less excitable in rats exposed to alcohol compared with controls.

\section{Materials and Methods}

All the experiments were conducted in accordance with the Society for Neuroscience Policies on the Use of Animals and Humans in Neuroscience Research.

Newborn Wistar rats [ethanol-treated (Et) group, three litters] received ethanol by inhalation from the second postnatal day (P2; P0 is the birthdate) through P6 (Fig. $1 A$ ). An air pump (air flow $=31 / \mathrm{min}$ ) was connected to a vaporization chamber into which ethanol $(95 \% \mathrm{v} / \mathrm{v})$ was injected at a rate of $2.5 \mathrm{ml} / \mathrm{min}$. The vaporization chamber was kept at the constant temperature of $38^{\circ} \mathrm{C}$. Ethanol vapors were conveyed to a Plexiglas cage in which the pups were placed, after separation from the mothers, for $3 \mathrm{~h}$ a day. A small fan circulated the air inside the cage, where the temperature was kept at $22-24^{\circ} \mathrm{C}$. This method of ethanol administration yields peak blood alcohol concentration ranging from 150 to 300 mg/dl (Granato et al., 2003; Granato, 2006). Control animals of matched age (Co group, three litters) were separated from the mothers for $3 \mathrm{~h}$ a day, omitting the ethanol inhalation procedure. To control for possible effects of hypothermia deriving from a synergy between alcohol exposure and maternal separation, either the alcohol exposure or the control procedure was performed without maternal separation in two additional litters, keeping the dam in the Plexiglas chamber.

In vitro electrophysiology was performed on pyramidal neurons in somatosensory neocortical slices from P30-P60 animals (Fig. 1B). We did not observe any obvious difference in the electrophysiological properties between male and female rats either in alcohol-treated or control rats. Rats were deeply anesthetized with halothane and decapitated. Parasagittal slices (300 $\mu \mathrm{m}$ thick) were cut in ice-cold, oxygenated solution 


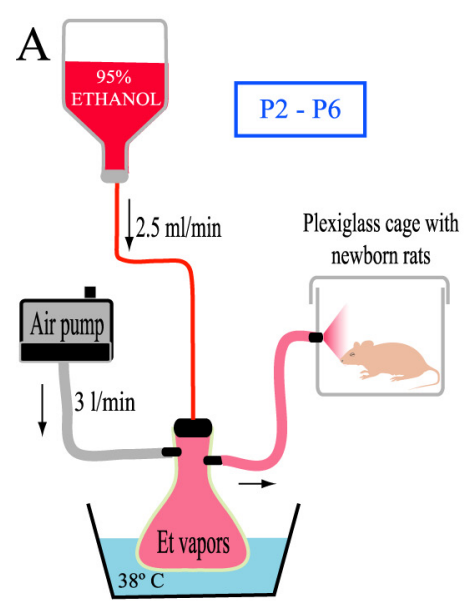

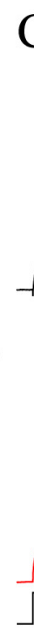
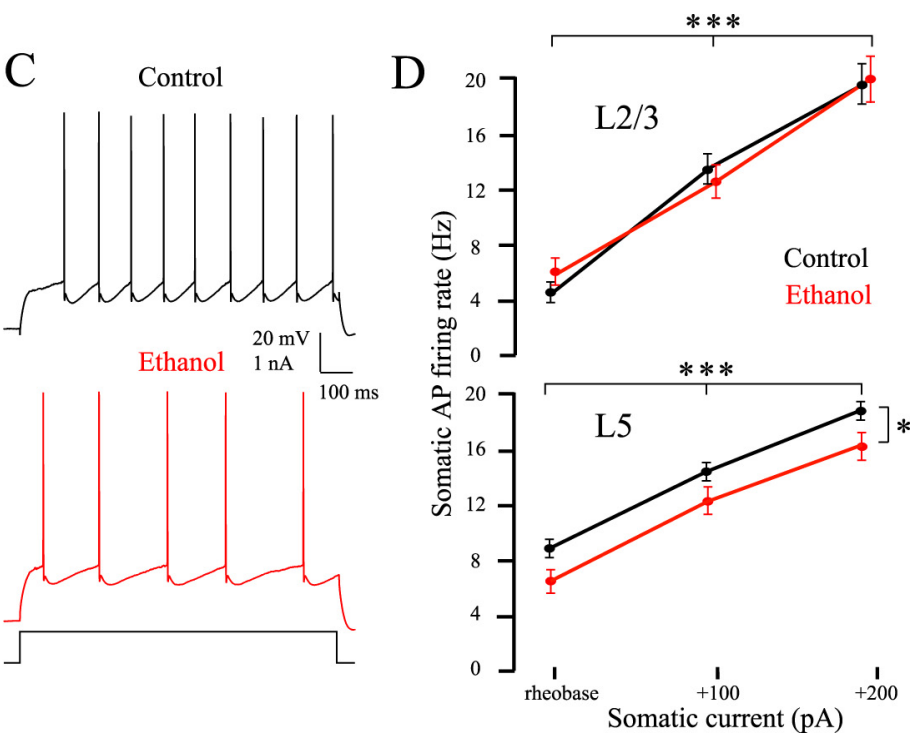

Figure 1. $\quad A, B$, Experimental procedure performed at $P 2-P 6$ (ethanol exposure, $A$ ) and during adulthood (slice recording, $B$ ). $C$, Somatic recording of representative $L 5$ neurons, following steps of depolarizing current injected at soma (rheobase for both neurons). $\boldsymbol{D}$, Somatic firing rate after somatic current injection. ${ }^{*} p<0.05 ;{ }^{* * *} p<0.001$.

containing the following (in mM): $125 \mathrm{NaCl}, 25 \mathrm{NaHCO}_{3}, 2.5 \mathrm{KCl}, 1.25$ $\mathrm{NaH}_{2} \mathrm{PO}_{4}, 1 \mathrm{MgCl}_{2}, 25$ glucose, $2 \mathrm{CaCl}_{2}$. Slices were maintained at $37^{\circ} \mathrm{C}$ in the above solution for $60 \mathrm{~min}$ and then at room temperature until use. Whole-cell somatic and dendritic current-clamp recordings were performed from visually identified layer 5 and layer $2 / 3$ pyramidal neurons using infrared differential interference contrast optics. Recording electrodes (soma: 6-10 M $\Omega$; dendrites: 10-25 $\mathrm{M} \Omega$ ) were made from borosilicate glass capillaries and used without further modifications. Dendritic recordings were aimed at the apical dendrite of layer 5 neurons at a distance ranging from 423 to $761 \mu \mathrm{m}$ from the soma. The intracellular solution contained the following (in $\mathrm{mM}$ ): $115 \mathrm{~K}$ gluconate, $20 \mathrm{KCl}$, 10 HEPES, $2 \mathrm{MgCl}_{2}$, 2 MgATP, $2 \mathrm{Na}_{2}$ ATP, $0.3 \mathrm{NaGTP}, 0.2 \%$ biocytin, $\mathrm{pH}$ 7.3. In some cases, $100 \mu \mathrm{M}$ Oregon Green 488 BAPTA-1 (Invitrogen) was added to the somatic pipette for calcium imaging. Recordings were performed at $34.0 \pm 0.5^{\circ} \mathrm{C}$ using BVC 700A amplifiers (Dagan). Data were acquired with an Instrutech ITC-18 board (Heka) and analyzed using Igor Pro software (Wavemetrics). All values are given as mean \pm SEM. Differences between groups were evaluated using the ANOVA for nested design. For the current-frequency curves, we used the ANOVA for mixed design (with the current level as within-subject and the treatment as between-subject factor).

\section{Results}

Four to 7 weeks after exposing the newborn rats to alcohol, pyramidal neurons of layer 5 (L5)b and layer 2/3 (L2/3) of the somatosensory cortex were selected for electrophysiological recordings (Fig. $1 \mathrm{~B}$ ). Cells were filled with biocytin during recordings for morphological analysis of their dendritic trees. As already demonstrated for L2/3 pyramidal neurons (Granato et al., 2003), the basal dendrites of L5 pyramidal neurons had a shorter total length in Et neurons than in Co neurons $(\mathrm{Co}=4889.68 \pm 306.91$ $\mu \mathrm{m} ; \mathrm{Et}=4113.86 \pm 233.48 \mu \mathrm{m} ; F_{(1,6)}=5.87, p=0.052 ; n=9$ Co, 17 Et neurons, from 3 and 5 animals, respectively). There was no significant difference in the total length of the apical tree between Et and Co neurons.

We tested the basic subthreshold and suprathreshold properties with long ( $1 \mathrm{~s})$ square pulses in increasing steps (increments of $100 \mathrm{pA}$ ) at the cell body. The subthreshold properties of L5 neurons from Co and Et animals are shown in Table 1. The resting membrane potential, input resistance, and membrane time constant were not significantly different in the two considered
Table 1. Main electrophysiological properties of layer 5 pyramidal neurons

\begin{tabular}{lllll}
\hline & Control & Ethanol & $F_{(1,10)}$ & $p$ \\
\hline Number of animals & 7 & 5 & & \\
Number of neurons & 19 & 19 & & \\
Resting membrane potential $(\mathrm{mV})$ & $-72.69 \pm 1.01$ & $-73.67 \pm 1.77$ & 0.02 & 0.905 \\
Input resistance (MS) & $23.31 \pm 1.79$ & $22.54 \pm 1.10$ & 0.00 & 0.964 \\
Membrane time constant (ms) & $11.43 \pm 0.84$ & $13.12 \pm 1.17$ & 0.53 & 0.482 \\
Rheobase (pA) & $726.32 \pm 43.15$ & $705.26 \pm 42.90$ & 0.46 & 0.512 \\
Absolute threshold (mV) & $-42.56 \pm 0.82$ & $-41.78 \pm 1.28$ & 0.02 & 0.892 \\
Spike amplitude (mV) & $87.46 \pm 0.75$ & $87.15 \pm 0.91$ & 0.09 & 0.775 \\
Spike half-width (ms) & $0.63 \pm 0.02$ & $0.72 \pm 0.04$ & 0.50 & 0.496 \\
Fast AHP (mV) & $-7.77 \pm 0.43$ & $-7.27 \pm 0.71$ & 0.09 & 0.772 \\
Slow AHP (mV) & $-12.86 \pm 0.56$ & $-11.25 \pm 0.54$ & 1.96 & 0.192 \\
\hline Sind & & &
\end{tabular}

Subthreshold and suprathreshold properties of $C 0$ and Et layer 5 pyramidal neurons, as observed after injection of $1 \mathrm{~s}$ steps of current at soma. AHP, Afterhyperpolarization.

groups. The threshold current for action potential (AP) firing (rheobase) was also the same in Et and Co neurons (Table 1). However, for any given suprathreshold current injection (Fig. $1 C, D)$, the firing frequency of L5 Et neurons was lower than that of Co neurons ( $n=19$ Co, 19 Et neurons, from 7 and 5 animals, respectively). Despite differences in firing frequency, the slope of the current-frequency curve was similar in neurons from controls and ethanol-treated animals, but the curve was significantly shifted to the right in Et animals (Alcohol treatment: $F_{(1,36)}=6.33, p<$ 0.05; Current level: $F_{(2,72)}=714.98, p<0.001$; Interaction: $F_{(2,72)}=0.281, p=0.756$; Fig. $\left.1 D\right)$. In addition to rheobase, absolute voltage threshold, AP duration, afterhyperpolarization (both fast and slow), and the spike amplitude were all similar in Co and Et neurons (Table 1). These results show that despite a general lack of influence on most subthreshold and suprathreshold properties as measured at the cell body, early exposure to alcohol led to a change in excitability of L5 neurons. As to L2/3 neurons, there were no significant differences in the subthreshold and suprathreshold properties between Co and Et neurons. Unlike L5 neurons, the current-frequency curve of L2/3 was similar in the two groups (Alcohol treatment: $F_{(1,30)}=0.09, p=0.770$; Current level: $F_{(2,60)}=261.34, p<0.001$; Interaction: $F_{(2,60)}=1.35$, $p=0.267 ; n=16 \mathrm{Co}, 16 \mathrm{Et}$ neurons, from 5 and 4 animals, respectively; Fig. $1 D)$. 

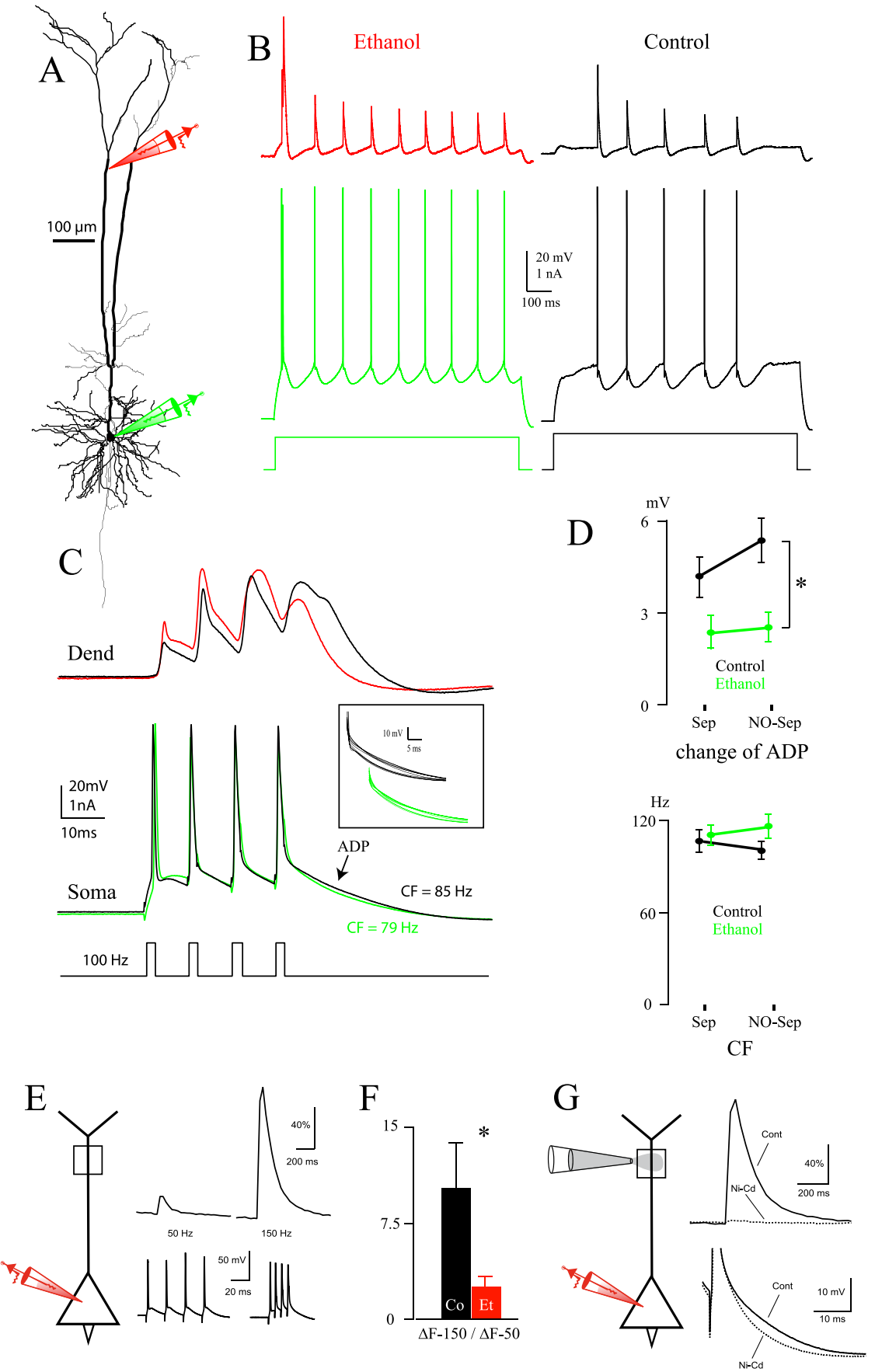

Figure 2. $\quad \boldsymbol{A}$, Reconstruction of an Etneuron in which dual somatic/dendritic recordings were performed. $\boldsymbol{B}$, Simultaneous dendritic and somatic recordings in representative $C 0$ and Et neurons after injection of $1 \mathrm{~s}$ steps of depolarizing current through the somatic pipette (Et neuron reconstructed in $\boldsymbol{A}$ ). The dendritic patch was performed at 761 and $670 \mu \mathrm{m}$ from soma in the $(0$ and Et neuron, respectively. $C$, High-frequency trains of spikes evoked by 2 ms somatic current pulses in the sameneurons shown in $\boldsymbol{B}(100 \mathrm{~Hz}$, above (F for both neurons). The backpropagating action potentials recorded at dendrites (Dend) are represented by black and red traces for the $\mathrm{C} 0$ and Et neuron, respectively. The somatic (Soma) APs are represented by black and green traces for the $\mathrm{C} 0$ and Et neuron, respectively. The inset shows superimposed traces of the ADP at frequencies ranging from $50 \mathrm{to} 130 \mathrm{~Hz}$. D, Change of ADP (occurring at CF) (top) and CF (bottom). Two-way ANOVA showed a significant effect of alcohol treatment on $\operatorname{ADP}\left({ }^{*} p<0.05\right)$, while there was no significant effect of the maternal separation protocol (Sep vs NO-Sep). E, Calcium imaging performed in a Co neuron at $800 \mu \mathrm{m}$ from the cell body (top traces) after somatic stimulation eliciting trains of four APs at 50 and $150 \mathrm{~Hz}$, respectively (bottom traces). Note the difference between $\Delta F$ at 50 and $150 \mathrm{~Hz}$. $F$, Ratio between $\Delta$ Fat $150 \mathrm{~Hz}$ and $\Delta$ Fat $50 \mathrm{~Hz}\left(800 \mu \mathrm{m}\right.$ from soma; $\left.{ }^{*}<0.05\right) . G,\left(\mathrm{Cd}^{2+}(50 \mu \mathrm{m})\right.$ and Ni ${ }^{2+}(100 \mu \mathrm{m})$ injected in proximity of the dendrite at $600 \mu \mathrm{m}$ from soma abolished the calcium transient (top traces) and the somatic ADP (bottom traces) normally elicited by somatic stimulation at $150 \mathrm{~Hz}$. Cont, Control.

The effect on spike firing in the absence of obvious changes in somatic properties suggested that dendritic ion channels might contribute to the integrative properties of the neuron leading to the lower firing frequency in L5 Et neurons. We therefore mea- sured the active properties in the dendrites of L5 neurons with dual somatic/ dendritic recordings (Fig. 2A). Current steps (1 s) applied at the soma evoked trains of APs that propagated back into the apical dendrite (Fig. $2 B$ ). In both Co and Et neurons, the amplitude of the backpropagating AP (bAP) decreased as a function of the distance of the recording electrode from the soma. After correcting for the distance from the soma, the ratio $\mathrm{bAP} /$ somatic AP was not significantly different, suggesting that alcohol treatment did not affect voltage-gated sodium channels along the apical dendrite $(\mathrm{Et}=0.48 \pm 0.08$; $\mathrm{Co}=0.59 \pm 0.03 ; n=10 \mathrm{Co}, 13$ Et neurons, from 5 and 5 animals, respectively; ANCOVA, with distance from the soma as covariate: $\left.F_{(1,20)}=1.75, p=0.201\right)$.

Dendritic calcium channels have also been shown to affect the firing properties of L5 pyramidal neurons (Larkum and Zhu, 2002). Using high-frequency trains of bAPs (Fig. 2C), the frequency at which dendritic calcium electrogenesis occurs [the critical frequency (CF)] can be determined from the afterdepolarization (ADP) at the soma (Larkum et al., 1999a, 2007). To exclude some of the alcohol effects that might be strengthened by maternal separation, these experiments were also performed in ethanol-treated and control animals not separated from their mothers. As shown in Figure 2D, neither the alcohol treatment nor the separation had a significant effect on the CF, and the interaction between treatment and separation was not significant (Alcohol treatment: $F_{(1,14)}=3.34, p=0.089$; Separation: $F_{(1,14)}=0.03, p=0.871$; Interaction: $F_{(1,14)}=0.25, p=0.627 ; n=26 \mathrm{Co}$ and 32 Et neurons, from 10 and 8 animals, respectively). In five of 32 ethanol-treated neurons (16\%) a CF was not evident, while only one of 26 control neurons did not display any $\mathrm{CF}$ $\left(\chi^{2}=2.15, p=0.143\right)$.

The CF represents the threshold for dendritic calcium electrogenesis through the accumulated superposition of bAPs in the dendrite. The similarity of the CF in ethanol-treated and control rats was therefore consistent with the fact that sodiumdependent propagation of APs along the dendrite was not altered. We were able to confirm this hypothesis directly with dendritic patch recordings $(n=10 \mathrm{Co}, 13 \mathrm{Et}$ neurons, from 6 and 5 animals, respectively). Here, the amplitude of the last bAP at supracritical frequencies was not significantly different between Et and Co neurons $(\mathrm{Co}=44.57 \pm 2.95 \mathrm{mV}$; $\left.\mathrm{Et}=37.47 \pm 3.50 \mathrm{mV} ; F_{(1,9)}=2.29, p=0.164\right)$. Interestingly, however, the duration of the last bAP was significantly shorter in Et than Co neurons at supracritical frequencies (half-width: Co $=$ 
$6.19 \pm 0.49 \mathrm{~ms} ; \mathrm{Et}=4.79 \pm 0.41 \mathrm{~ms} ; F_{(1,9)}$ $=5.77, p<0.05)$. The resulting change of $\mathrm{ADP}$ at the soma was significantly smaller in amplitude in ethanol-treated animals (both separated and nonseparated) than in control animals (Alcohol treatment: $F_{(1,14)}=$ 5.95, $p<0.05$; Separation: $F_{(1,14)}=1.41$, $p=0.254$; Interaction: $F_{(1,14)}=0.73, p=$ 0.406; Fig. $2 D)$. Altogether, results of Figure $2 A-D$ suggest that although there is no effect on bAPs, alcohol treatment does indeed have an effect on dendritic electrogenesis. Calcium imaging experiments confirmed that in control neurons, at a distance of 600-800 $\mu \mathrm{m}$ from the soma, the calcium initiation zone was characterized by a weak calcium signal after somatic stimulation at $50 \mathrm{~Hz}$. Conversely, the signal increased significantly when the somatic stimulation was at $150 \mathrm{~Hz}$ (i.e., above CF; Fig. 2 E). As shown in Figure $2 F$, this gain in calcium entry, expressed as the ratio between $\Delta F$ at $150 \mathrm{~Hz}$ and $\Delta F$ at $50 \mathrm{~Hz}$, was significantly lower for Et than for Co animals $\left(F_{(1,6)}=7.70, p<\right.$ $0.05 ; n=3 \mathrm{Co}, 5$ Et neurons, from 3 and 5 animals, respectively). Local application of $50 \mu \mathrm{M} \mathrm{Cd}{ }^{2+}$ and $100 \mu \mathrm{M} \mathrm{Ni}^{2+}$ close to the calcium initiation zone completely abolished the calcium transient elicited by somatic stimulation at $150 \mathrm{~Hz}$ (Fig. 2G). The somatic ADP was also abolished, therefore confirming that $\mathrm{ADP}$ is a faithful readout of calcium electrogenesis occurring at the distal apical dendrite (Fig. $2 G$ ).

We further tested the effect of alcohol treatment on dendritic electrogenesis by applying direct current injection into the apical dendrite (Fig. 3A). Whereas $1 \mathrm{~s}$ square pulses at the soma evoked trains of regularly spiking APs, the same square pulses injected into the dendrite in Co neurons generated somatic burst spiking and dendritic spikes with a prominent calcium component (Fig. 3B) (Larkum and Zhu, 2002). Conversely, most Et neurons displayed fewer and shorter dendritic spikes, suggesting a substantial decrease in the calcium component. Furthermore, these decreased dendritic spikes were often accompanied by only a single somatic AP (Fig. 3B). As shown in Figure 3C, the quantitative parameters confirmed the impairment of calcium spikes in dendrites of ethanol-treated animals: the mean duration and the number of dendritic spikes were lower in Et than in Co neurons, and the difference was significant for the duration (half-width 100 pA above rheobase: $\mathrm{Co}=42.23 \pm 10.07 \mathrm{~ms}$; Et $=$ $11.84 \pm 3.58 \mathrm{~ms} ; F_{(1,16)}=4.64, p<0.05 ; n=19 \mathrm{Co}, 12 \mathrm{Et}$ neurons, from 11 and 7 animals, respectively). The distribution of half-widths of dendritic spikes confirms this finding, showing that the majority of Et neurons displayed dendritic spikes with half-widths shorter than $10 \mathrm{~ms}$ (Fig. 3D). The rheobase for eliciting dendritic spikes was not significantly different in Co and Et controls (black traces).

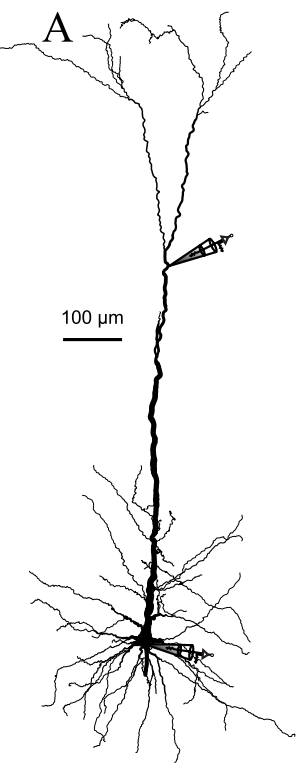

C

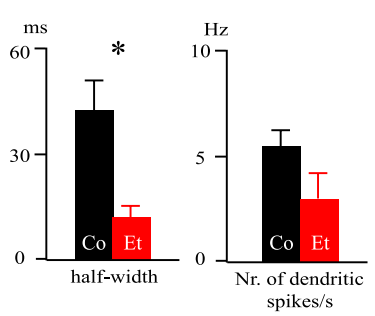

$\mathrm{E}$

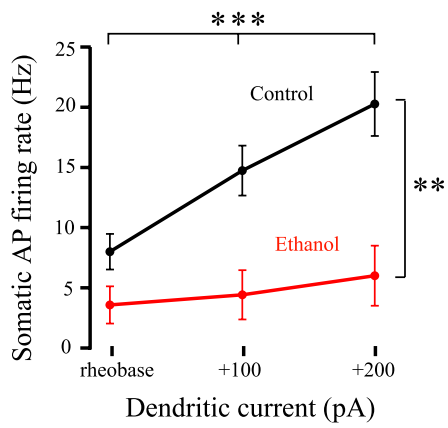

B
Control Ethanol

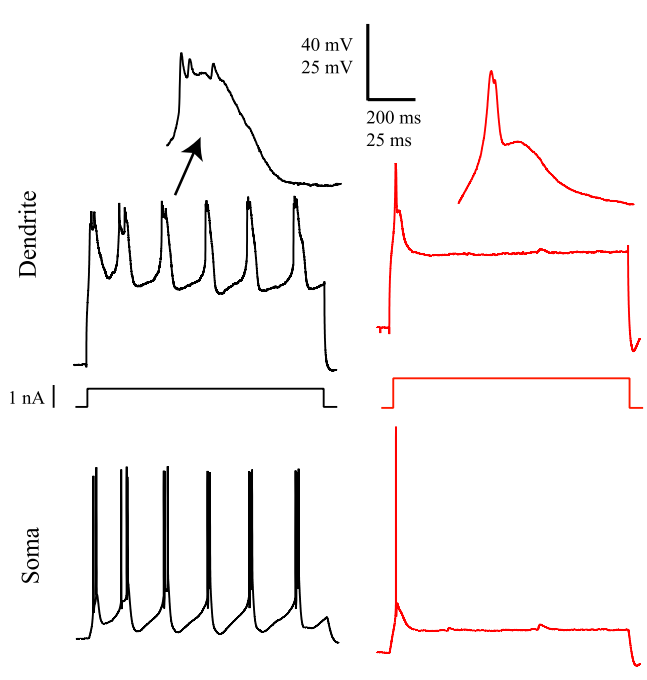

D

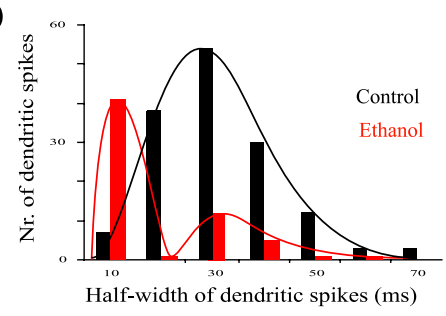

$\mathrm{F}$

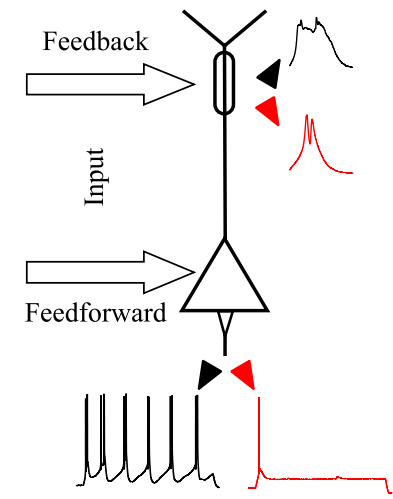

Figure 3. A, Reconstruction of a Co neuron in which dual somatic/dendritic recordings were performed. $B$, Simultaneous dendritic and somatic recordings in representative $C 0$ and Et neurons after injection of $1 \mathrm{~s}$ steps of depolarizing current through the dendritic pipette (100 pA above rheobase; Co neuron reconstructed in $A$ ). The dendritic patch was performed at 617 and $620 \mu \mathrm{m}$ from soma in the $C 0$ and Et neuron, respectively. Two dendritic spikes are represented on a magnified time and voltage scale. $C$, Half-width and number of dendritic spikes per second following dendritic current injections $100 \mathrm{pA}$ above rheobase; ${ }^{*} p<0.05 . \boldsymbol{D}$, Frequency distribution of the half-width of dendritic spikes in $19 \mathrm{C} 0$ and 12 Et neurons. Data at rheobase and with current injection $100 \mathrm{pA}$ above rheobase were pooled. $\boldsymbol{E}$, Somatic firing rate after dendritic current injection; ${ }^{* *} p<0.01,{ }^{* * *} p<0.001$. $\boldsymbol{F}$, Summary diagram showing the main results emerging from the present data. The electrogenesis at the distal apical dendrite and, consequently, the firing pattern of the output are both strongly impaired after early exposure to alcohol (red traces) compared with

neurons $(\mathrm{Co}=983.33 \pm 50.65 \mathrm{pA} ; \mathrm{Et}=958.33 \pm 79.26 \mathrm{pA} ;$ $\left.F_{(1,16)}=0.01, p=0.923\right)$. Finally, the number of somatic spikes elicited through $1 \mathrm{~s}$ steps of dendritic current was significantly lower in Et than in Co neurons at rheobase and 100 and $200 \mathrm{pA}$ above rheobase (Treatment: $F_{(1,25)}=8.71, p<0.01$; Current level: $F_{(2,50)}=33.76, p<0.001$; Interaction: $F_{(2,50)}=17.26, p<$ $0.001 ; n=19$ Co, 12 Et neurons, from 11 and 7 animals; Fig. $3 E$ ). The divergence in the slopes of the dendritic current/somatic frequency curves (confirmed by the highly significant statistical 
interaction) suggests that the gain of pyramidal neurons (Larkum et al., 2004) is dramatically reduced after early exposure to ethanol.

\section{Discussion}

We have shown that early exposure to ethanol leads to a permanent downregulation of dendritic electrogenesis in L5 pyramidal neurons. Direct dendritic patch recordings indicated that an impairment of dendritic calcium signaling is responsible for the observed alterations. It is known that alcohol inhibits voltagegated calcium channels in developing hippocampal pyramidal neurons (Zucca and Valenzuela, 2010). The acute effects reported by Zucca and Valenzuela (2010) would have to be maintained in young adult animals several weeks after the end of alcohol exposure. What could be the possible mechanism to explain the long-lasting observed effects?

Early alcohol exposure leads to several alterations of cortical microcircuits involving the corticocortical associative connections (Granato et al., 2003), as well as the number, distribution, and molecular properties of GABAergic interneurons (Granato, 2006; Tavian et al., 2011). As a consequence, the cortical network activity could display striking alterations, which could in turn influence the expression, targeting, or modulation of ion channels. Preliminary RT-PCR experiments from our group, performed on homogenates of the somatosensory cortex, indicate that there is an underexpression of Cav1.2 subunit transcripts in alcohol-treated rats (data not shown). These L-type channels have been hypothesized to account for a large part of dendritic calcium spikes (Almog and Korngreen, 2009), and their block by nimodipine in hippocampal pyramidal neurons leads to a reduction of ADP and bursting, similar to that observed in our study (Magee and Carruth, 1999). However, we cannot rule out that other calcium channels and/or mechanisms related to their functional properties could also be involved. It is known that voltagegated channel trafficking is regulated by neuronal activity (Kim et al., 2007). Pozzi et al. (2008) demonstrated that the network activity can modulate neuronal voltage-gated calcium channels through the induction of protein phosphorylation. Interestingly, Servais et al. (2007) observed that calcium entry into cerebellar Purkinje cells was reduced after early exposure to alcohol. Servais et al. (2007) attributed this alteration to a reduced phosphorylation of voltage-gated calcium channels.

The functional consequences of the reduced dendritic calcium spikes observed in ethanol-treated animals remain to be established. The supralinear calcium influx in the distal apical dendrite is thought to enable pyramidal neurons to associate ascending sensory inputs and the long-range feedback input to the superficial layers (Larkum et al., 1999b). Our experiments show that early alcohol exposure has a dramatic effect on the output of pyramidal neurons in response to distal dendritic input. We therefore would predict that cortical processing dependent on feedback information in pyramidal neurons would be significantly impaired (Fig. $3 F$ ). In addition, calcium spikes are critically involved in the genesis and modulation of spike-timing-dependent plasticity for distal synapses (Letzkus et al., 2006). It is worth mentioning that Meredith et al. (2007) studied the synaptic plasticity in a genetic model of mental retardation, i.e., the Fmr1 knock-out mice, a well established reproduction of Fragile X syndrome. Meredith et al. (2007) demonstrated an increased threshold for spike-timingdependent plasticity in cortical pyramidal neurons of knock-out mice and showed that this is the consequence of compromised calcium signaling in spines and dendrites.
Layer 5 pyramidal neurons represent an important source of output from the neocortex. Therefore, the reduced rate of somatic APs that we observed in alcohol-treated animals, both during somatic and dendritic current injection, could have great functional relevance. Clearly, a complex electrical remodeling occurs in Et neurons due to downregulation of dendritic voltagesensitive ion channels that no longer participate in the integrative process and leave the cell generally less excitable. In this focused study, we did not attempt to show that the reduced firing rate of L5 pyramidal neurons represents the electrophysiological counterpart of the cognitive defects of FASD. In fact, very few studies have addressed this issue. In a rat model of mental retardation due to congenital hypothyroidism, Sánchez-Alonso et al. (2010) noted that hippocampal pyramidal neurons showed a lower number of spikes per burst. The authors demonstrated that the mechanism was related to a reduction in the afterdepolarization, which we also observed. This reduction in burst firing may be responsible for a degradation in the transmission of salient information (Lisman, 1997). Last, in their pivotal review, Kaufmann and Moser (2000) assert that "dendritic anomalies are the most consistent anatomical correlates of mental retardation." It is therefore plausible that the effects shown in our study might contribute to the cognitive deficits seen in FASD. Their further elucidation could open totally new therapeutical approaches to mental retardation.

\section{References}

Almog M, Korngreen A (2009) Characterization of voltage-gated Ca(2+) conductances in layer 5 neocortical pyramidal neurons from rats. PLoS One 4:e4841.

Dobbing J, Sands J (1979) Comparative aspects of the brain growth spurt. Early Hum Dev 3:79-83.

Granato A (2006) Altered organization of cortical interneurons in rats exposed to ethanol during neonatal life. Brain Res 1069:23-30.

Granato A, Di Rocco F, Zumbo A, Toesca A, Giannetti S (2003) Organization of cortico-cortical associative projections in rats exposed to ethanol during early postnatal life. Brain Res Bull 60:339-344.

Guerri C, Bazinet A, Riley EP (2009) Foetal alcohol spectrum disorders and alterations in brain and behaviour. Alcohol Alcohol 44:108-114.

Ikonomidou C, Bittigau P, Ishimaru MJ, Wozniak DF, Koch C, Genz K, Price MT, Stefovska V, Hörster F, Tenkova T, Dikranian K, Olney JW (2000) Ethanol-induced apoptotic neurodegeneration and fetal alcohol syndrome. Science 287:1056-1060.

Kaufmann WE, Moser HW (2000) Dendritic anomalies in disorders associated with mental retardation. Cereb Cortex 10:981-991.

Kim J, Jung SC, Clemens AM, Petralia RS, Hoffman DA (2007) Regulation of dendritic excitability by activity-dependent trafficking of the A-type $\mathrm{K}+$ channel subunit Kv4.2 in hippocampal neurons. Neuron 54:933-947.

Larkum ME, Zhu JJ (2002) Signaling of layer 1 and whisker-evoked Ca2+ and $\mathrm{Na}+$ action potentials in distal and terminal dendrites of rat neocortical pyramidal neurons in vitro and in vivo. J Neurosci 22:6991-7005.

Larkum ME, Kaiser KM, Sakmann B (1999a) Calcium electrogenesis in distal apical dendrites of layer 5 pyramidal cells at a critical frequency of back-propagating action potentials. Proc Natl Acad Sci USA 96:14600-14604.

Larkum ME, Zhu JJ, Sakmann B (1999b) A new cellular mechanism for coupling inputs arriving at different cortical layers. Nature 398:338-341.

Larkum ME, Senn W, Lüscher HR (2004) Top-down dendritic input increases the gain of layer 5 pyramidal neurons. Cereb Cortex 14:1059-1070.

Larkum ME, Waters J, Sakmann B, Helmchen F (2007) Dendritic spikes in apical dendrites of neocortical layer $2 / 3$ pyramidal neurons. J Neurosci 27:8999-9008.

Letzkus JJ, Kampa BM, Stuart GJ (2006) Learning rules for spike timingdependent plasticity depend on dendritic synapse location. J Neurosci 26:10420-10429.

Lisman JE (1997) Bursts as a unit of neural information: making unreliable synapses reliable. Trends Neurosci 20:38-43.

Magee JC, Carruth M (1999) Dendritic voltage-gated ion channels regulate 
the action potential firing mode of hippocampal CA1 pyramidal neurons. J Neurophysiol 82:1895-1901.

Medina AE, Krahe TE (2008) Neocortical plasticity deficits in fetal alcohol spectrum disorders: lessons from barrel and visual cortex. J Neurosci Res $86: 256-263$.

Meredith RM, Holmgren CD, Weidum M, Burnashev N, Mansvelder HD (2007) Increased threshold for spike-timing-dependent plasticity is caused by unreliable calcium signaling in mice lacking fragile $\mathrm{X}$ gene FMR1. Neuron 54:627-638.

Miller MW, Chiaia NL, Rhoades RW (1990) Intracellular recording and injection study of corticospinal neurons in the rat somatosensory cortex: effect of prenatal exposure to ethanol. J Comp Neurol 297:91-105.

Mooney SM, Miller MW (2001) Effects of prenatal exposure to ethanol on the expression of bcl-2, bax and caspase 3 in the developing rat cerebral cortex and thalamus. Brain Res 911:71-81.

Pozzi D, Condliffe S, Bozzi Y, Chikhladze M, Grumelli C, Proux-Gillardeaux V, Takahashi M, Franceschetti S, Verderio C, Matteoli M (2008) Activity-dependent phosphorylation of Ser187 is required for SNAP-25negative modulation of neuronal voltage-gated calcium channels. Proc Natl Acad Sci U S A 105:323-328.
Sánchez-Alonso JL, Muñoz-Cuevas J, Vicente-Torres MA, Colino A (2010) Role of low-voltage-activated calcium current on the firing pattern alterations induced by hypothyroidism in the rat hippocampus. Neuroscience 171:993-1005.

Servais L, Hourez R, Bearzatto B, Gall D, Schiffmann SN, Cheron G (2007) Purkinje cell dysfunction and alteration of long-term synaptic plasticity in fetal alcohol syndrome. Proc Natl Acad Sci U S A 104:9858-9863.

Tavian D, De Giorgio A, Granato A (2011) Selective underexpression of Kv3.2 and Kv3.4 channels in the cortex of rats exposed to ethanol during early postnatal life. Neurol Sci 32:571-577.

Xie N, Yang Q, Chappell TD, Li CX, Waters RS (2010) Prenatal alcohol exposure reduces the size of the forelimb representation in motor cortex in rat: an intracortical microstimulation (ICMS) mapping study. Alcohol 44:185-194.

Zucca S, Valenzuela CF (2010) Low concentrations of alcohol inhibit BDNF-dependent GABAergic plasticity via L-type Ca2 + channel inhibition in developing CA3 hippocampal pyramidal neurons. J Neurosci 30: 6776-6781. 